Nenad Petrović

Vesna Marjanović

Nenad Kostić $\square$

Nenad Marjanović

Mircea Viorel Dragoi

https://doi.org/10.21278/TOF.44303

ISSN 1333-1124

eISSN 1849-1391

\title{
MEANS AND EFFECTS OF CONSTRAINING THE NUMBER OF USED CROSS-SECTIONS IN TRUSS SIZING OPTIMIZATION
}

\begin{abstract}
Summary
This paper looks at sizing optimization results, and attempts to show the practical implications of using a novel constraint. Most truss structural optimization problems, which consider sizing in order to minimize weight, do not consider the number of different crosssections that the optimal solution can have. It was observed that all, or almost all, crosssections were different when conducting the sizing optimization. In practice, truss structures have a small, manageable number of different cross-sections. The constraint of the number of different cross-sections, proposed here, drastically increases the complexity of solving the problem. In this paper, the number of different cross-sections is limited, and optimization is done for four different sizing optimization problems. This is done for every number of different cross-section profiles which is smaller than the number of cross-sections in the optimal solution, and for a few numbers greater than that number. All examples are optimized using dynamic constraints for Euler buckling and discrete sets of cross-section variables. Results are compared to the optimal solution without a constrained number of different crosssections and to an optimal model with just a single cross-section for all elements. The results show a small difference between optimal solutions and the optimal solutions with a limited number of different profiles which are more readily applicable in practice.
\end{abstract}

Key words: $\quad$ truss optimization, cross-sections, Euler buckling, sizing, optimization constraints

\section{Introduction}

In recent years, structural optimization in general has evolved from being a tool for finding optimal solutions to specific parts of engineering problems to basically giving a completely developed design ready for manufacturing, thanks to improvements in the $\mathrm{CAD} / \mathrm{CAM} / \mathrm{CAE}$ software and the use of intricate optimization methods. Structural optimization of trusses is a complex real-world problem which must consider many different aspects in defining a mathematical model in order to achieve results which can be used for the construction. The most commonly optimized aspect of a truss is the bar cross-section selection, or sizing optimization, as it is called in the literature. 
Research in the field of truss structural optimization has lately become mostly focused on the use of novel methods to solve standard problems and show improvements in the algorithm. A lot of studies published to date, which use various optimization methods for sizing optimization, consider cross-section variables as continuous. Bekdas et al. [1] used a flower pollination algorithm to achieve competitive results in continuous sizing optimization, having tested their algorithm on numerous standard truss problems. Researchers in [2] presented a new method for size optimization of trusses; they compared their continuous results to discrete and continuous solutions from the literature. In [3], a heuristic method to achieve optimal weight was developed, using sizing of standard 10, 18, 72, and 200-bar trusses with continuous variables. Researchers in [4-6] also used continuous variables for sizing and showed the improved convergences of their methods. Kanarachos et al. [7] developed an optimization algorithm for efficient truss optimization and tested it on a continuous and a discrete variable cross-section problem of a 25-bar truss.

Since continuous variables for cross-section areas are not a realistic representation of what is possible in practice, researchers have also considered the use of discrete variables. This approach, though it appears to be simpler, actually drastically increases the difficulty with which the method finds an optimal solution as the search space is discontinuous, nonlinear, non-convex and implicit with respect to design variables.

Hasancebi and K. Azad [8] developed an adaptive dimensional search algorithm specifically for discrete truss sizing optimization. Their research considers fixed slenderness ratios for tension and compression members as well as stress and displacement constraints. In their research, Cheng et al. [9] presented a variant of the harmony search algorithm for solving discrete sizing optimization and tested it on numerous truss problems without buckling constraints.

A few studies which consider dynamic buckling constraints have been published in recent years [10-15]. In [12], the authors made a comparison between using and not using buckling constraints for truss sizing problems with continuous variables. In their research, they also tested existing results from the literature which do not consider this constraint and found that all models have some bar elements which would not meet buckling criteria.

The inflation of novel optimization methods in the literature has led research away from improving the way engineering problems are solved in this field, and has focused it on incremental improvements in the used algorithm performance. This is the case in most aforementioned publications. In an attempt to achieve optimal results which have a practical value, this research considers the use of buckling constraints with discrete sizing variables.

Based on a research overview and personal experience, it has been found that the number of different cross-sections used in optimal solutions is very high. A large number of different cross-sections is impractical for many reasons. Namely, the number of different profiles of stock to be purchased and cut to size is large and leaves a lot of wasted material; in addition, there are problems caused by the onsite assembly with a great possibility of human error. This paper aims to introduce a novel constraint for sizing optimization which would limit the maximum number of different cross-sections used in the optimal solution. Such a constraint dramatically increases the complexity of the problem. As there are no other studies dealing with this constraint, in this paper, a comparison between optimal results for typical truss sizing optimization problems and optimal models limited to reasonable numbers of different cross-sections. This is done to show the influence of decreasing the number of different cross-sections on optimal weight, and to find a recommended value for the constraint. 


\section{Truss sizing optimzation: problem formulation and examples}

In order to achieve applicable results, the truss sizing optimization problem must use an optimization method which can handle a large number of variables and search spaces. The problem becomes even greater when considering all necessary constraints which further complicate finding the global optima. In order to verify the effectiveness of introducing new constraints, the process has to be tested on comparable examples frequently found in the literature.

\subsection{Optimization}

Optimization is the process of finding solutions from a group of possible solutions to a given, mathematically defined, problem. Such solutions are difficult or impossible to find in other ways; these solutions provide favourable characteristics while decreasing the invested effort and cost. As heuristic optimization methods are able to work with a large number of variables, can overcome local extremes, are very fast and efficient, and have a low threshold of needed inputs about the problem to find the solution, they are favoured for solving engineering optimization problems. For the purpose of this research, a genetic algorithm (GA) [16] is used due to its favourable characteristics and availability. This research does not consider comparisons between algorithm performances.

Truss sizing optimization uses cross-sectional geometrical dimensions as variables. Our research looks at cross-section variables as a discrete set of values. The objective is to find a combination of cross-sections which gives a minimal weight. Typical truss sizing problems found in the literature view the minimal weight design problem as follows:

$$
\left\{\begin{array}{l}
\min W(A)=\sum_{i=1}^{i=n} \rho_{i} A_{i} l_{i} \text { with } A=\left(A_{1}, \ldots, A_{n}\right) \\
\text { subjected to }\left\{\begin{array}{l}
A_{\min } \leq A_{i} \leq A_{\max } \text { for } i=1, \ldots, n \\
\sigma_{\min } \leq \sigma_{i} \leq \sigma_{\max } \text { for } i=1, \ldots, n \\
u_{\min } \leq u_{j} \leq u_{\max }
\end{array} \text { for } j=1, \ldots, k\right.
\end{array}\right.
$$

where $W$ is the weight of the truss, $n$ is the number of truss elements, $k$ is the number of nodes, $l_{i}$ is the length of the $i^{\text {th }}$ element, $A_{i}$ is the area of the $i^{\text {th }}$ element cross-section, $\sigma_{i}$ is the stress of the $i^{\text {th }}$ element, and $u_{j}$ is the displacement of the $j^{\text {th }}$ node.

In order to achieve results which can be applied in practice, constraints for Euler buckling are added to the optimization problem. As the Euler critical buckling load equation (3) has a change in the moment of inertia in each iteration, due to the change in cross-sections, this constraint is considered to be dynamic. The addition of this constraint increases the complexity of the optimization problem significantly. In the expression for Euler buckling, (2), the same areas figure as denominators on both sides of the expression; therefore, the critical force load (3) can be used as the buckling constraint to minimize calculation. Then, the constraint is used as given in (4).

$$
\begin{aligned}
& \sigma_{A i}^{c o m p} \leq \sigma_{K i} \\
& \text { where } \sigma_{A i}^{c o m p}=\frac{F_{A i}^{c o m p}}{A_{i}} \text { and } \sigma_{K i}=\frac{F_{K i}}{A_{i}} \\
& F_{K i}=\frac{\pi^{2} \cdot E_{i} \cdot I_{i}}{l_{i}^{2}} \\
& \left|F_{A i}^{c o m p}\right| \leq F_{K i} \text { for } i=1, \ldots, n
\end{aligned}
$$


where $\sigma_{A i}$ is the axial compression stress of the $i^{\text {th }}$ bar element, and $\sigma_{K i}$ is the critical buckling stress of the $i^{\text {th }}$ element; $F_{A i}{ }^{\text {comp }}$ is the axial compression force, $F_{K i}$ is Euler's critical load of the $i^{\text {th }}$ element, $E_{i}$ is the $i^{\text {th }}$ element's modulus of elasticity and $I_{i}$ is the minimum area moment of inertia of the $i^{\text {th }}$ element's cross-section. The condition from equation (4) is added to the existing constraints from equation (1).

Experience has shown that the use of sizing optimization gives models with a large number of different cross-sections, which is hard to manage in practice. As stock is available in fixed lengths, it is desirable if more than one piece for a bar element can be cut from a single piece of stock. Having to purchase a large number of different cross-sections of stock is therefore potentially more expensive and wasteful. With the goal of achieving the most practically applicable model from optimization, the authors of this paper suggest the use of a new constraint which limits the number of different cross-sections used. Such a constraint increases the complexity of solving the already multi-modal discontinuous function. The mathematical formulation for this constraint is given as:

$$
\begin{gathered}
\left|\left\{A_{1}^{G}, A_{2}^{G}, A_{3}^{G}, \ldots, A_{n}^{G}\right\}\right|=m \\
m \leq m_{\max }
\end{gathered}
$$

where $A_{n}^{G}$ is the cross-section geometry area of the $n^{\text {th }}$ element, $m$ is the cardinal of the crosssection area geometry set, and $m_{\max }$ is the maximal allowed number of different crosssections. In the original software developed for the purpose of this research, two variable sets were created for sizing optimization. The first set includes $m$ variables which can each adopt a cross-section diameter from the list of available profiles. The second set of variables assigns the cross-sections to each bar from the previous set of variables. This way, the constraint set by (5) is always satisfied. In this paper, in order to see the influence of limiting the number of different cross-sections, the first set of variables was forced to have all different cross-sections selected, and the second was forced to have at least one of each different cross-section from the set. This means that the inequality in expression (5) is set to an equality, and that there are $m_{\max }$ different first variables.

This type of constraint has never been used in papers published to date. This research aims to show the influence of adding such a constraint to $2 \mathrm{D}$ and $3 \mathrm{D}$ truss examples depending on the number of different cross-sections allowed. Some of the most frequently used sizing optimization problems in the literature are 10,17, and 25-bar truss problems. These examples were previously analysed in [12], where the authors, using the same models with continuous cross-section variables, showed the influence of adding the Euler buckling constraints. The addition of the Euler buckling constraints to truss structural optimization problems ensures that the optimal truss configurations can stay in the elastic zone and maintain stability. This research will use discrete values for cross-section areas as well as buckling and other constraints given in the examples.

\subsection{Examples}

A 10-bar truss problem setup and the node layout are shown in Figure 1. Truss elements are made of aluminium 6063-T5, whose characteristics are: Young modulus of $68947 \mathrm{MPa}$ and density of $2.7 \mathrm{~g} / \mathrm{cm}^{3}$. Two load cases are tested, the first load case (LC1) has point loads of $P_{l}=444.82 \mathrm{kN}, P_{2}=0 \mathrm{kN}$, and the second load case (LC2) has point loads of $P_{l}=667.233 \mathrm{kN}$ and $P_{2}=222.411 \mathrm{kN}$, Figure 1. Optimization is limited with a maximal displacement of $\pm 0.0508 \mathrm{~m}$ of all nodes in all directions, axial stress of $\pm 172.3689 \mathrm{MPa}$ for all bars, and the Euler buckling constraints for all bars. A discrete set of variables for full round cross-sections made of aluminium 6063-T5 was compiled from available standard dimensions acquired from several vendors. There are 50 possible cross-section profile diameters ranging from $12 \mathrm{~mm}$ $\left(1.131 \mathrm{~cm}^{2}\right)$ to $356 \mathrm{~mm}\left(995.382 \mathrm{~cm}^{2}\right)$. 
A 17-bar truss problem setup and the node layout are shown in Figure 2. Material characteristics for all elements are: Young modulus of $206842.719 \mathrm{MPa}$, and density of $7.4 \mathrm{~g} / \mathrm{cm}^{3}$. A load of $444.82 \mathrm{kN}$ is applied in node 9, as shown in Figure 2. The optimization is constrained by a displacement limitation of $\pm 0.0508 \mathrm{~m}$ for all nodes, in both $\mathrm{x}$ and $\mathrm{y}$ directions, and by the Euler buckling constraints for all bars. This example does not have a fixed stress constraint. A discrete set of variables for full round cross-sections of this steel was compiled from available standard dimensions acquired from several vendors. There are 49 possible cross-section profile diameters ranging from $6 \mathrm{~mm}\left(0.283 \mathrm{~cm}^{2}\right)$ to $250 \mathrm{~mm}$ $\left(490.894 \mathrm{~cm}^{2}\right)$.

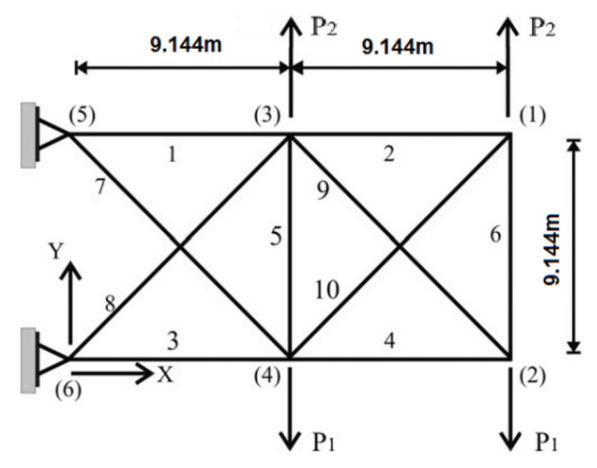

Fig. 1 10-bar truss problem setup

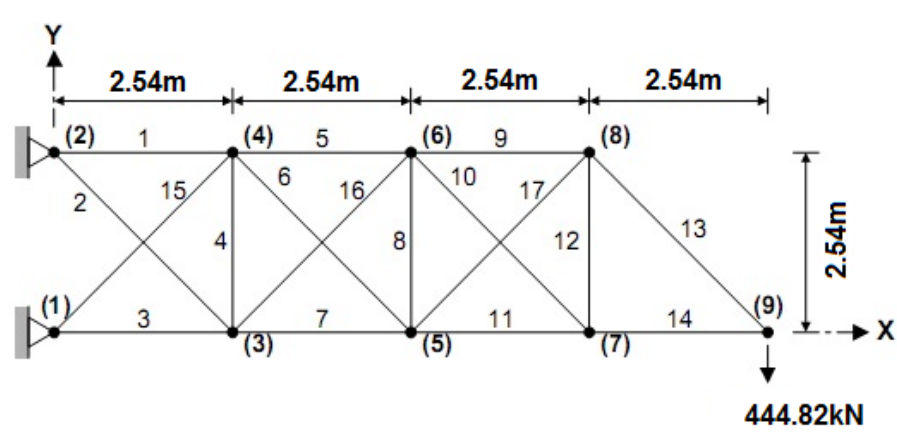

Fig. 2 17-bar truss problem setup

A 25-bar truss problem setup and the node layout are shown in Figure 3. Material characteristics for all elements are the same as for the 10-bar truss example, as well as the cross-section variable set. Force vectors in nodes are as follows: node 1 (4.448, -44.48, $44.48) \mathrm{kN}$, node $2(0,-44.48,-44.48) \mathrm{kN}$, node $3(2.224,0,0) \mathrm{kN}$, and node $6(2.6688,0,0)$ $\mathrm{kN}$. The space truss cross-sections are grouped as follows: $1\left(\mathrm{~A}_{1}\right), 2\left(\mathrm{~A}_{2}-\mathrm{A}_{5}\right), 3\left(\mathrm{~A}_{6}-\mathrm{A}_{9}\right)$, $4\left(\mathrm{~A}_{10}-\mathrm{A}_{11}\right), 5\left(\mathrm{~A}_{12}-\mathrm{A}_{13}\right), 6\left(\mathrm{~A}_{14}-\mathrm{A}_{17}\right), 7\left(\mathrm{~A}_{18}-\mathrm{A}_{21}\right)$, and $8\left(\mathrm{~A}_{22}-\mathrm{A}_{25}\right)$. Optimization is limited by a tensile stress limit of $40 \mathrm{kN}$ for all bar groups and a maximal displacement of $\pm 0.00889 \mathrm{~m}$ for all nodes in all directions.

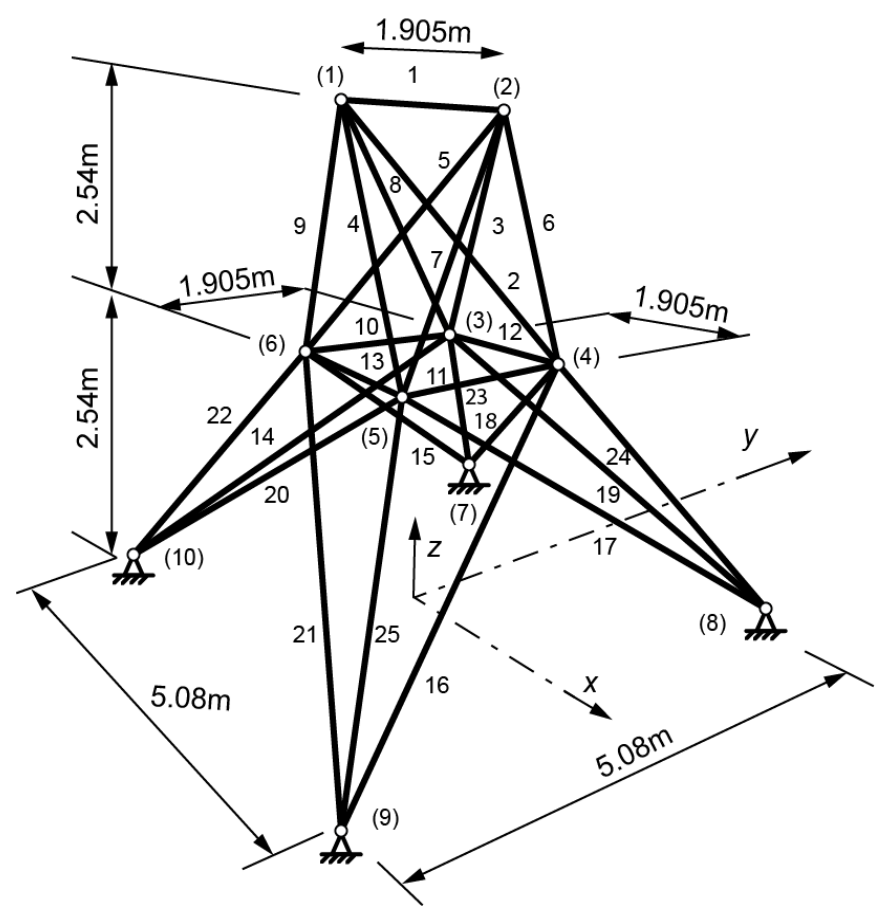

Fig. 3 25-bar truss problem setup 
N. Petrović, V. Marjanović, N. Kostić,

N. Marjanović, M. V. Dragoi
Means and Effects of Constraining the Number of Used Cross-Sections in Truss Sizing Optimization

\section{Results}

As conventional optimal solutions give a large number of different cross-sections, results of each limited optimal model are compared to both the optimal solution and a solution which uses only one cross-section.

For the 10-bar truss model, the optimal weights of limited models and their differences from the optimal solution and single cross-section models are given in Tables 1 and 3 for load cases 1 and 2, respectively.

Table 1 Optimal weights and differences for the 10-bar truss problem LC1

\begin{tabular}{|c|c|c|c|}
\hline $\begin{array}{c}\text { No. of different cross- } \\
\text { sections }\end{array}$ & Weight / kg & $\begin{array}{c}\text { Difference from the solution with a } \\
\text { single cross-section }\end{array}$ & Difference from the optimal solution \\
\hline 1 & 13089.261 & - & $172.936 \%$ \\
\hline 2 & 6752.918 & $48.409 \%$ & $40.811 \%$ \\
\hline 3 & 5924.592 & $54.737 \%$ & $23.539 \%$ \\
\hline 4 & 5643.872 & $56.882 \%$ & $17.685 \%$ \\
\hline 5 & 5552.247 & $57.582 \%$ & $15.775 \%$ \\
\hline 6 & 5292.524 & $59.566 \%$ & $10.359 \%$ \\
\hline 7 & 5251.098 & $59.882 \%$ & $9.495 \%$ \\
\hline 8 & 4795.734 & $63.361 \%$ & - \\
\hline 9 & 5223.248 & $60.095 \%$ & $8.914 \%$ \\
\hline 10 & 5506.26 & $57.933 \%$ & $14.816 \%$ \\
\hline
\end{tabular}

The cross-section areas of all bars for each model are given in Tables 2 and 4, for load cases 1 and 2 , respectively.

Table 2 Cross-section areas of optimal models for the 10-bar truss problem LC1

\begin{tabular}{|c|c|c|c|c|c|c|c|c|c|c|}
\hline \multirow{2}{*}{ Bar number } & \multicolumn{6}{|c|}{ Cross-section areas / $\mathrm{cm}^{2}$ for models constrained to specific numbers of different cross-sections } \\
\cline { 2 - 11 } & 1 & 2 & 3 & 4 & 5 & 6 & 7 & 8 & 9 & 10 \\
\hline 1 & 452.389 & 452.389 & 452.389 & 452.389 & 415.476 & 415.476 & 415.476 & 78.540 & 415.476 & 452.389 \\
\hline 2 & 452.389 & 452.389 & 314.159 & 201.062 & 201.062 & 153.938 & 153.938 & 15.904 & 181.458 & 153.938 \\
\hline 3 & 452.389 & 78.540 & 78.540 & 78.540 & 78.540 & 23.758 & 23.758 & 415.475 & 33.183 & 23.758 \\
\hline 4 & 452.389 & 78.540 & 78.540 & 78.540 & 78.540 & 44.179 & 63.617 & 240.528 & 113.097 & 38.485 \\
\hline 5 & 452.389 & 78.540 & 78.540 & 78.540 & 78.540 & 113.097 & 113.097 & 1.131 & 86.590 & 113.097 \\
\hline 6 & 452.389 & 78.540 & 78.540 & 78.540 & 78.540 & 23.758 & 23.758 & 15.904 & 7.069 & 28.274 \\
\hline 7 & 452.389 & 78.540 & 78.540 & 78.540 & 78.540 & 113.097 & 95.033 & 122.718 & 86.590 & 122.719 \\
\hline 8 & 452.389 & 78.540 & 78.540 & 78.540 & 78.540 & 113.097 & 95.033 & 415.476 & 103.869 & 103.869 \\
\hline 9 & 452.389 & 452.389 & 452.389 & 452.389 & 452.389 & 380.133 & 380.133 & 103.869 & 380.133 & 380.1327 \\
\hline 10 & 452.389 & 452.389 & 314.159 & 314.159 & 314.159 & 380.133 & 380.133 & 181.458 & 346.361 & 415.476 \\
\hline Weight /kg & 13089.261 & 6752.918 & 5924.592 & 5643.872 & 5552.247 & 5245.509 & 5251.098 & 4795.734 & 5223.248 & 5506.26 \\
\hline
\end{tabular}

For the 17-bar truss model, the optimal weights of limited models and their differences from the optimal solution and single cross-section models are given in Table 5. Table 6 shows the optimal cross-section areas for the bars. 
Means and Effects of Constraining the Number of Used Cross-Sections in Truss Sizing Optimization
N. Petrović, V. Marjanović, N. Kostić, N. Marjanović, M. V. Dragoi

Table 3 Optimal weights and differences for the 10-bar truss problem LC2

\begin{tabular}{|c|c|c|c|}
\hline $\begin{array}{c}\text { No. of different } \\
\text { cross-sections }\end{array}$ & Weight $/ \mathrm{kg}$ & $\begin{array}{c}\text { Difference from the solution with a } \\
\text { single cross-section }\end{array}$ & Difference from the optimal solution \\
\hline 1 & 13089.26 & - & $211.954 \%$ \\
\hline 2 & 6623.126 & $49.400 \%$ & $57.848 \%$ \\
\hline 3 & 5630.351 & $56.985 \%$ & $34.187 \%$ \\
\hline 4 & 5258.692 & $59.824 \%$ & $25.329 \%$ \\
\hline 5 & 4977.002 & $61.976 \%$ & $18.616 \%$ \\
\hline 6 & 4508.238 & $65.558 \%$ & $7.444 \%$ \\
\hline 7 & 4442.876 & $66.057 \%$ & $5.886 \%$ \\
\hline 8 & 4263.527 & $67.427 \%$ & $1.612 \%$ \\
\hline 9 & 4229.672 & $67.686 \%$ & $0.805 \%$ \\
\hline 10 & 4195.899 & $67.944 \%$ & - \\
\hline
\end{tabular}

Table 4 Cross-section areas of optimal models for the 10-bar truss problem LC2

\begin{tabular}{|c|c|c|c|c|c|c|c|c|c|c|}
\hline \multirow{2}{*}{ Bar number } & \multicolumn{10}{|c|}{ Cross-section areas $/ \mathrm{cm}^{2}$ for models constrained to specific numbers of different cross-sections } \\
\hline & 1 & 2 & 3 & 4 & 5 & 6 & 7 & 8 & 9 & 10 \\
\hline 1 & 452.389 & 452.389 & 452.389 & 415.476 & 415.476 & 452.389 & 452.389 & 380.133 & 415.476 & 415.476 \\
\hline 2 & 452.389 & 452.389 & 226.980 & 254.469 & 226.980 & 283.529 & 283.529 & 283.529 & 254.469 & 254.469 \\
\hline 3 & 452.389 & 452.389 & 56.745 & 56.745 & 56.745 & 103.869 & 86.590 & 86.590 & 63.617 & 63.617 \\
\hline 4 & 452.389 & 70.882 & 56.745 & 56.745 & 33.183 & 9.621 & 9.079 & 9.079 & 12.566 & 12.566 \\
\hline 5 & 452.389 & 70.882 & 226.980 & 103.869 & 103.869 & 70.882 & 70.882 & 70.882 & 122.718 & 122.718 \\
\hline 6 & 452.389 & 70.882 & 56.745 & 56.745 & 33.183 & 33.183 & 28.274 & 28.274 & 3.142 & 3.142 \\
\hline 7 & 452.389 & 70.882 & 56.745 & 56.745 & 56.745 & 33.183 & 28.274 & 28.274 & 33.183 & 33.183 \\
\hline 8 & 452.389 & 70.882 & 56.745 & 103.869 & 103.869 & 103.869 & 113.097 & 113.097 & 103.869 & 103.869 \\
\hline 9 & 452.389 & 452.389 & 226.980 & 415.476 & 415.476 & 452.389 & 452.389 & 452.389 & 314.159 & 314.159 \\
\hline 10 & 452.389 & 70.882 & 452.389 & 254.469 & 226.980 & 9.621 & 9.079 & 9.079 & 122.718 & 113.097 \\
\hline Weight / kg & 13089.261 & 6623.126 & 5630.351 & 5258.692 & 4977.002 & 4508.238 & 4442.876 & 4263.527 & 4229.672 & 4195.899 \\
\hline
\end{tabular}

Table 5 Optimal weights and differences for the 17-bar truss problem

\begin{tabular}{|c|c|c|c|}
\hline $\begin{array}{c}\text { No. of different } \\
\text { cross-sections }\end{array}$ & Weight / kg & $\begin{array}{c}\text { Difference from the solution with a } \\
\text { single cross-section }\end{array}$ & Difference from the optimal solution \\
\hline 1 & 3181.777 & $35.653 \%$ & $30.250 \%$ \\
\hline 2 & 2047.368 & $42.296 \%$ & $16.803 \%$ \\
\hline 3 & 1836.005 & $44.222 \%$ & $12.905 \%$ \\
\hline 4 & 1774.722 & $46.822 \%$ & $7.643 \%$ \\
\hline 5 & 1692.007 & $47.354 \%$ & $6.566 \%$ \\
\hline 6 & 1675.086 & $50.585 \%$ & $0.026 \%$ \\
\hline 7 & 1572.28 & $50.598 \%$ & - \\
\hline 8 & 1571.875 & $46.228 \%$ & $8.844 \%$ \\
\hline 10 & 1710.898 & $46.936 \%$ & $7.412 \%$ \\
\hline
\end{tabular}


N. Petrović, V. Marjanović, N. Kostić,

N. Marjanović, M. V. Dragoi
Means and Effects of Constraining the Number of Used Cross-Sections in Truss Sizing Optimization

Table 6 Cross-section areas of optimal models for the 17-bar truss problem

\begin{tabular}{|c|c|c|c|c|c|c|c|c|c|c|}
\hline \multirow{2}{*}{ Bar number } & \multicolumn{7}{|c|}{ Cross-section areas / $\mathrm{cm}^{2}$ for models constrained to specific numbers of different cross-sections } \\
\cline { 2 - 12 } & 1 & 2 & 3 & 4 & 5 & 6 & 7 & 8 & 9 & 10 \\
\hline 1 & 86.590 & 44.179 & 44.179 & 44.179 & 44.179 & 44.179 & 44.179 & 44.179 & 44.179 & 44.179 \\
\hline 2 & 86.590 & 44.179 & 44.179 & 44.179 & 23.758 & 23.758 & 23.758 & 23.758 & 23.758 & 23.758 \\
\hline 3 & 86.590 & 86.590 & 86.590 & 86.590 & 86.590 & 86.590 & 86.590 & 86.590 & 95.033 & 95.033 \\
\hline 4 & 86.590 & 44.179 & 44.179 & 44.179 & 23.758 & 23.758 & 0.503 & 0.503 & 23.758 & 11.341 \\
\hline 5 & 86.590 & 44.179 & 44.179 & 44.179 & 56.745 & 56.745 & 56.745 & 56.745 & 38.485 & 38.485 \\
\hline 6 & 86.590 & 44.179 & 44.179 & 44.179 & 23.758 & 23.758 & 23.758 & 23.758 & 23.758 & 23.758 \\
\hline 7 & 86.590 & 86.590 & 86.590 & 86.590 & 86.590 & 86.590 & 86.590 & 86.590 & 86.590 & 86.590 \\
\hline 8 & 86.590 & 44.179 & 44.179 & 38.485 & 23.758 & 23.758 & 0.283 & 0.283 & 0.283 & 0.503 \\
\hline 9 & 86.590 & 86.590 & 44.179 & 38.485 & 38.485 & 38.485 & 38.485 & 38.485 & 78.540 & 78.540 \\
\hline 10 & 86.590 & 44.179 & 44.179 & 38.485 & 38.485 & 38.485 & 38.485 & 38.485 & 38.485 & 38.485 \\
\hline 11 & 86.590 & 86.590 & 56.745 & 56.745 & 56.745 & 56.745 & 56.745 & 56.745 & 78.540 & 78.540 \\
\hline 12 & 86.590 & 44.179 & 44.179 & 38.485 & 38.485 & 38.485 & 38.485 & 38.485 & 38.485 & 38.485 \\
\hline 13 & 86.590 & 44.179 & 44.179 & 38.485 & 38.485 & 38.485 & 38.485 & 38.485 & 38.485 & 38.485 \\
\hline 14 & 86.590 & 44.179 & 44.179 & 44.179 & 44.179 & 44.179 & 44.179 & 44.179 & 44.179 & 44.179 \\
\hline 15 & 86.590 & 44.179 & 44.179 & 44.179 & 56.745 & 50.265 & 50.265 & 50.265 & 50.265 & 50.265 \\
\hline 16 & 86.590 & 44.179 & 44.179 & 44.179 & 56.745 & 56.745 & 56.745 & 56.745 & 50.265 & 50.265 \\
\hline 17 & 86.590 & 86.590 & 56.745 & 56.745 & 56.745 & 56.745 & 50.265 & 50.265 & 56.745 & 56.745 \\
\hline Weight / $\mathrm{kg}$ & 3181.777 & 2047.368 & 1836.005 & 1774.722 & 1692.007 & 1675.086 & 1572.28 & 1571.875 & 1710.898 & 1688.375 \\
\hline
\end{tabular}

For the 25-bar space truss model, the optimal weights of limited models and their differences from the optimal solution and single cross-section models are given in Table 7. Table 8 shows the optimal cross-section areas for the bar groups according to the number of different cross-sections of the model.

Table 7 Optimal weights and differences for the 25-bar truss problem

\begin{tabular}{|c|c|c|c|}
\hline No. of different cross-sections & Weight / kg & $\begin{array}{c}\text { Difference from the solution } \\
\text { with a single cross-section }\end{array}$ & $\begin{array}{c}\text { Difference from the optimal } \\
\text { solution }\end{array}$ \\
\hline 1 & 1007.379 & - & $46.611 \%$ \\
\hline 2 & 819.530 & $18.647 \%$ & $19.272 \%$ \\
\hline 3 & 770.276 & $23.537 \%$ & $12.104 \%$ \\
\hline 4 & 736.686 & $26.871 \%$ & $7.215 \%$ \\
\hline 5 & 703.108 & $30.204 \%$ & $2.328 \%$ \\
\hline 6 & 702.455 & $30.269 \%$ & $2.233 \%$ \\
\hline 7 & 697.573 & $30.754 \%$ & $1.523 \%$ \\
\hline 8 & 687.111 & $31.792 \%$ & - \\
\hline
\end{tabular}

The differences in weight from the optimal solution for each limited number of different cross-sections, for all examples, are shown in Figure 4 with an expanded view in order to better illustrate the values. 
Table 8 Bar group cross-section areas of optimal models for the 25-bar truss problem

\begin{tabular}{|c|c|c|c|c|c|c|c|c|c|c|}
\hline \multirow{2}{*}{ Bar group } & \multicolumn{7}{|c|}{ Cross-section areas / $\mathrm{cm}^{2}$ for models constrained to specific numbers of different cross-section groups } \\
\cline { 2 - 13 } & 1 & 2 & 3 & 4 & 5 & 6 & 7 & 8 & 9 & 10 \\
\hline 1 & 44.179 & 33.183 & 44.179 & 4.909 & 3.142 & 1.131 & 1.131 & 1.131 & 44.179 & 33.183 \\
\hline 2 & 44.179 & 33.183 & 44.179 & 33.183 & 23.758 & 28.274 & 23.758 & 23.758 & 44.179 & 33.183 \\
\hline 3 & 44.179 & 33.183 & 33.183 & 28.274 & 38.485 & 33.183 & 33.183 & 33.183 & 44.179 & 33.183 \\
\hline 4 & 44.179 & 33.183 & 4.909 & 4.909 & 3.142 & 1.131 & 1.131 & 2.011 & 44.179 & 33.183 \\
\hline 5 & 44.179 & 33.183 & 4.909 & 33.183 & 3.142 & 4.909 & 15.904 & 4.909 & 44.179 & 33.183 \\
\hline 6 & 44.179 & 33.183 & 33.183 & 28.274 & 28.274 & 28.274 & 28.274 & 28.274 & 44.179 & 33.183 \\
\hline 7 & 44.179 & 33.183 & 33.183 & 33.183 & 38.485 & 38.485 & 38.485 & 38.485 & 44.179 & 33.183 \\
\hline 8 & 44.179 & 50.265 & 44.179 & 50.265 & 44.179 & 44.179 & 44.179 & 44.179 & 44.179 & 50.265 \\
\hline Weight/ kg & 1007.379 & 819.530 & 770.276 & 736.686 & 703.108 & 702.455 & 697.573 & 687.111 & 1007.379 & 819.530 \\
\hline
\end{tabular}

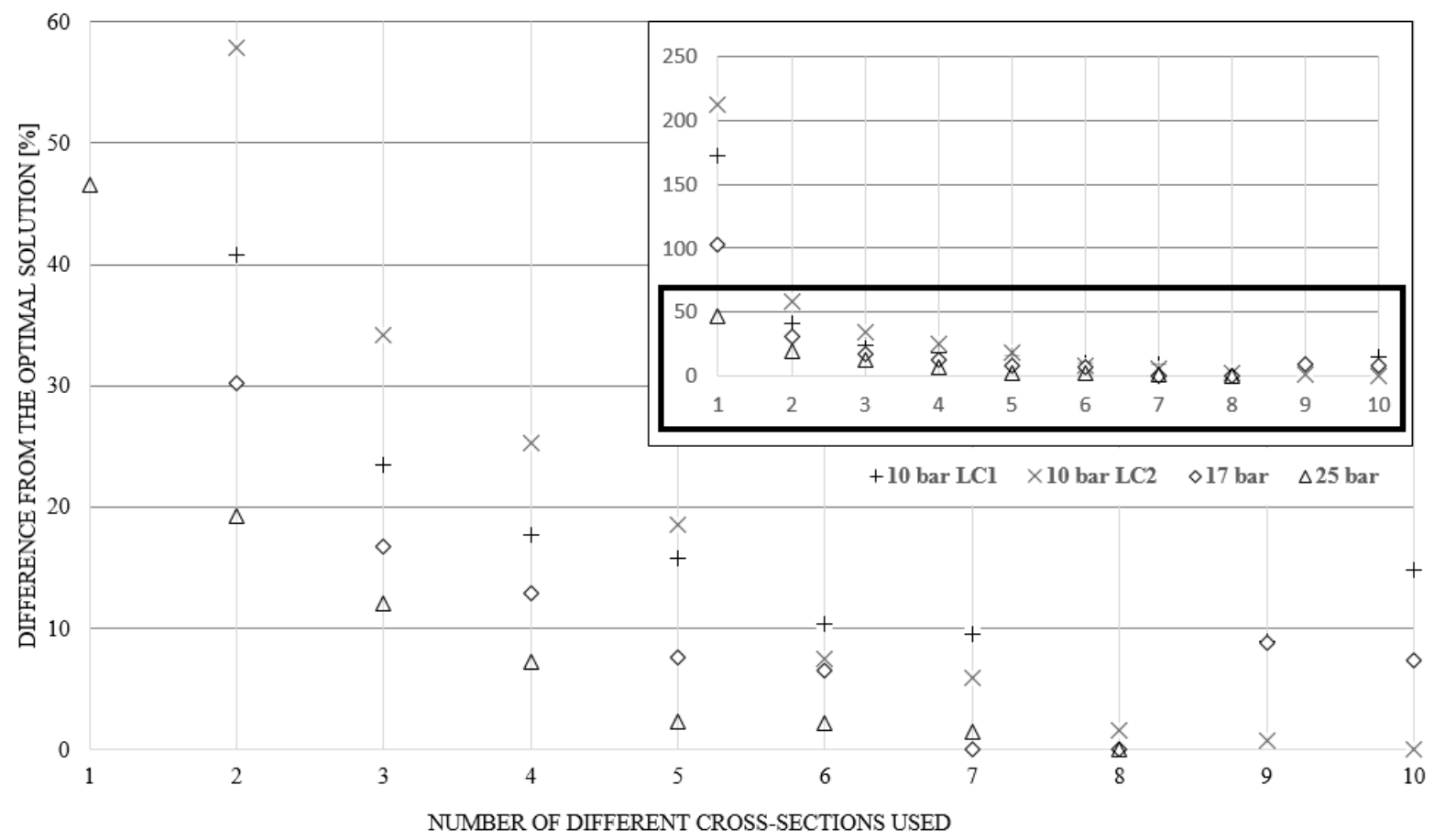

Fig. 4 Differences from optimal solutions without a limited number of cross-sections used.

All optimizations were conducted a number of times, and the best solutions for each setup were selected as representative choices in the results. It should be noted that due to the increased complexity of the added forced constraints, convergence was difficult, and the termination criteria for the optimization method had to be considered in order to avoid local optima. The termination criterion was therefore set as a maximum stagnant population. The optimization method used was a genetic algorithm, GA, due to the availability of the software and the abundance of use in the literature.

Results show a substantial decrease in weight in all models when going from using only a single cross-section to using two different sizes. This is, namely, due to the influence of the Euler buckling constraint which warrants larger cross-section areas for bars subjected to axial compressive forces. By precisely determining the number of different cross-sections which can be used, the results presented here were much more difficult to optimize. For the 17-bar truss, the examples were only run for a maximum number of 10 different cross-sections, as the optimal number of 8 different cross-sections is already a large number. It can be seen that 
once the optimal number of cross-sections is exceeded, the weight of the optimized models begins to increase again. This is due to the fact that the model must use larger, available crosssections where it would normally use the same areas as with another bar which is similarly loaded.

\section{Conclusion}

Most research studies published in recent years in the field of truss structural optimization are focused on optimization algorithm performances. In engineering practice, designed trusses use a few different cross-sections. In the literature, the sizing optimization generally gives optimal results without limiting the number of used cross-section sizes; this result in structures which use a large, impractical, number of different cross-section profiles. In this paper, examples of planar 10 and 17-bar trusses, as well as of a 25-bar space truss, were optimized using discrete sizing variables. In addition, buckling constraints were added to those of the standard examples in order to ensure structural integrity. The new constraint, which limits the number of cross-section profiles that the optimization can use in a single solution, is intended for making the results of truss design optimization directly applicable in practice.

The 10-bar truss problem was optimized for two different load cases. The results show that the optimal number of different cross-sections for the sizing optimization of these trusses is 8 or more. This corresponds to other examples of discrete sizing optimization solutions found in the literature. In practice, one to four different cross-sections would be used on these types of constructions because an increased number of different bar cross-sections increases the complexity of the calculations and assembly, as well as potential costs due to a larger amount of unused stock. This research presents the optimal results obtained using the novel constraint which limits the number of different cross-section profiles that the optimal solution can have.

Two differences were considered in this research in terms of the weight difference with respect to the number of different cross-section profiles used. The first is the difference in weight from the solution with a single profile, and the other is the difference from the optimal solution without a limited number of different cross-section profiles. For the 10-bar truss in LC1, an optimal weight of $4795.734 \mathrm{~kg}$ is achieved with 8 different cross-sections, or $63.361 \%$ less than with the single profile model. In LC2, the optimal weight is achieved with all bars using different cross-sections; the weight is $4195.899 \mathrm{~kg}$, which is $67.944 \%$ less than with the single profile model. The 17-bar truss gives an optimal weight of $1571.875 \mathrm{~kg}$, with 8 different cross-section profiles without the new constraint, which is $50.598 \%$ less than with the single cross-section profile model. The 25-bar truss is specific not only because it is a space truss, but also because the bars are grouped. The optimal weight for this model is $687.111 \mathrm{~kg}$ with all 8 different cross-section profile group areas, giving a model $31.792 \%$ lighter than its single profile counterpart.

In order to determine how much heavier the models with a limited number of crosssections would be, the optimal mass of the models with a limited cross-section were compared to the optimal solution without this constraint. For the 10-bar truss in LC1, the model with 6 different cross-section profiles has $\sim 10 \%(10.359 \%)$ greater weight, while that with 3 different cross-section profiles has a weight which is greater by $\sim 20 \%(23.539 \%)$ than the optimum, where 8 different cross-section profiles are used. For the 10-bar truss in LC2, the model with 6 different cross-section profiles has $<10 \%(7.444 \%)$ greater weight and that with 5 different cross-section profiles $<20 \%(18.616 \%)$ greater weight than the optimum, where 10 different cross-section profiles are used. The 17-bar truss with 4 different crosssection profiles has $<10 \%(7.643 \%)$ greater weight, while that with 3 different profiles has a 
weight which is greater by $<20 \%(12.905 \%)$ than the optimum, where 8 different crosssection profiles are used. The 25-bar truss with 3 different cross-section profile bar groups weighs by $\sim 10 \%$ (12.104\%) more, while that with 2 different cross-section profiles used in bar groups weighs by $<20 \%(19.272 \%)$ more than the optimal model, where 8 different crosssection profile groups are used.

The approximate $10 \%$ and $20 \%$ differences from the optimum without the constrained number of different cross-sections were used in order to find the average number of different cross-section profiles which are used within this range; subsequently, it is used to determine a recommendation for the $m_{\max }$ constraint from expression (3). The average number of different cross-section profiles used in this range is 4 . This being a reasonable number for practical application, the authors recommend its use in sizing optimization problems as a new constraint in order to achieve optimal solutions which are more practically applicable.

\section{Acknowledgment}

This paper is a result of the projects TR32036 "Software development for solving coupled multiphysics problems", and TR33015 "Investigation and development of Serbian zero-net energy house". We would like to thank the Ministry of Education, Science and Technological Development of the Republic of Serbia for the financial support during this research.

\section{REFERENCES}

[1] G. Bekdaş, S.M. Nigdeli, X.-S. Yang, Sizing optimization of truss structures using flower pollination algorithm, Applied Soft Computing, 37 (2015) 322-331. https://doi.org/10.1016/j.asoc.2015.08.037

[2] A. Kaveh, R. Sheikholeslami, S. Talatahari, M. Keshvari-Ilkhichi, Chaotic swarming of particles: A new method for size optimization of truss structures, Advances in Engineering Software, 67 (2014) 136-147. https://doi.org/10.1016/j.advengsoft.2013.09.006

[3] M. Khatibinia, H. Yazdani, Accelerated multi-gravitational search algorithm for size optimization of truss structures, Swarm and Evolutionary Computation, 38 (2018) 109-119. https://doi.org/10.1016/j.swevo.2017.07.001

[4] S.O. Degertekin, M.S. Hayalioglu, Sizing truss structures using teaching-learning-based optimization, Computers \& Structures, 119 (2013) 177-188. https://doi.org/10.1016/j.compstruc.2012.12.011

[5] T. Dede, S. Bekiroğlu, Y. Ayvaz, Weight minimization of trusses with genetic algorithm, Applied Soft Computing, 11 (2011) 2565-2575. https://doi.org/10.1016/j.asoc.2010.10.006

[6] T. Xu, W. Zuo, T. Xu, G. Song, R. Li, An adaptive reanalysis method for genetic algorithm with application to fast truss optimization, Acta Mechanica Sinica, 26 (2009) 225-234. https://doi.org/10.1007/s10409-009-0323-x

[7] S. Kanarachos, J. Griffin, M.E. Fitzpatrick, Efficient truss optimization using the contrast-based fruit fly optimization algorithm, Computers \& Structures, 182 (2017) 137-148. https://doi.org/10.1016/j.compstruc.2016.11.005

[8] O. Hasançebi, S.K. Azad, Adaptive dimensional search: A new metaheuristic algorithm for discrete truss sizing optimization, Computers \& Structures, 154 (2015) 1-16. https://doi.org/10.1016/j.compstruc.2015.03.014

[9] M.-Y. Cheng, D. Prayogo, Y.-W. Wu, M.M. Lukito, A Hybrid Harmony Search algorithm for discrete sizing optimization of truss structure, Automation in Construction, 69 (2016) 21-33. https://doi.org/10.1016/j.autcon.2016.05.023

[10] V.J. Savsani, G.G. Tejani, V.K. Patel, P. Savsani, Modified meta-heuristics using random mutation for truss topology optimization with static and dynamic constraints, Journal of Computational Design and Engineering, 4 (2017) 106-130. https://doi.org/10.1016/j.jcde.2016.10.002

[11] A. Kaveh, V.R. Mahdavi, Colliding bodies optimization for size and topology optimization of truss structures, Structural Engineering and Mechanics, 53 (2015) 847-865. https://doi.org/10.12989/sem.2015.53.5.847

[12] N. Petrovic, N. Marjanovic, N. Kostic, M. Blagojevic, M. Matejic, S. Troha, Effects of introducing dynamic constraints for buckling to truss sizing optimization problems, FME Transaction, 46 (2018) 117123. https://doi.org/10.5937/fmet1801117p 
N. Petrović, V. Marjanović, N. Kostić,

N. Marjanović, M. V. Dragoi
Means and Effects of Constraining the Number of Used Cross-Sections in Truss Sizing Optimization

[13] N. Petrović, N. Kostić, N. Marjanović, COMPARISON OF APPROACHES TO 10 BAR TRUSS STRUCTURAL OPTIMIZATION WITH INCLUDED BUCKLING CONSTRAINTS, Applied Engineering Letters, 2 (2017) 98-103.

[14] H. Madah, O. Amir, Truss optimization with buckling considerations using geometrically nonlinear beam modeling, Computers \& Structures, 192 (2017) 233-247. https://doi.org/10.1016/j.compstruc.2017.07.023

[15] H. Ozbasaran, solveTruss v1.0: Static, global buckling and frequency analysis of 2D and 3D trusses with Mathematica, SoftwareX, 6 (2017) 135-140. https://doi.org/10.1016/j.softx.2017.05.004

[16] E.G. Shopova, N.G. Vaklieva-Bancheva, BASIC - A genetic algorithm for engineering problems solution, Computers \& Chemical Engineering, 30 (2006) 1293-1309.

https://doi.org/10.1016/j.compchemeng.2006.03.003

Submitted: $\quad 24.01 .2019$

Accepted: $\quad 02.12 .2019$
Nenad Petrović

Vesna Marjanović

Nenad Kostić (corresponding author)

Nenad Marjanović

University of Kragujevac, Faculty of

Engineering, Sestre Janjić 6, 34000

Kragujevac, Serbia

Mircea Viorel Dragoi

Universitatea Transilvania Brasov,

Bulevardul Eroilor 29, Brașov 500036,

Romania 